\title{
RECENT BCP PROGRESS IN TAIWAN
}

\author{
HSIANG-NAN LI \\ Department of Physics, National Cheng-Kung University, Tainan, Taiwan 701, \\ Republic of China \\ Physics Division, National Center for Theoretical Sciences, Hsinchu, Taiwan 300, \\ Republic of China

\begin{abstract}
I review theoretical progresses on $B$ physics and $\mathrm{CP}$ violation which were made in Taiwan recently. I concentrate on the approaches to exclusive $B$ meson decays based on factorization assumption, $S U(3)$ symmetry, perturbative QCD factorization theorem, QCD factorization, and light-front QCD formalism.
\end{abstract}

\section{Introduction}

The collaboration on $B$ physics and $\mathrm{CP}$ violation $(\mathrm{BCP})$ is one of the most active groups in Taiwan. In this talk I will briefly review theoretical progresses on BCP, which were made in Taiwan recently. For experimental review, please refer to Dr. Wang's talk in this workshop. I will concentrate on five approaches to exclusive $B$ meson decays based on factorization assumption (FA), $S U(3)$ symmetry, perturbative QCD (PQCD) factorization theorem, QCD factorization (QCDF), and light-front QCD (LFQCD) formalism. Abundant results of exclusive $B$ meson decays have been produced and important dynamics has been explored.

The effective Hamiltonian for $b$ quark decays, for example, the $b \rightarrow s$ transition, is given by

$$
H_{\mathrm{eff}}=\frac{G_{F}}{\sqrt{2}} \sum_{q=u, c} V_{q}\left[C_{1}(\mu) O_{1}^{(q)}(\mu)+C_{2}(\mu) O_{2}^{(q)}(\mu)+\sum_{i=3}^{10} C_{i}(\mu) O_{i}(\mu)\right],
$$

with the Cabibbo-Kobayashi-Maskawa (CKM) matrix elements $V_{q}=V_{q s}^{*} V_{q b}$. Using the unitarity condition, the CKM matrix elements for the penguin operators $O_{3}-O_{10}$ can also be expressed as $V_{u}+V_{c}=-V_{t}$. The definitions of the operators $O_{i}$ are referred tol.

According to the Wolfenstein parametrization, the CKM matrix upto $\mathcal{O}\left(\lambda^{3}\right)$ is written as

$$
\left(\begin{array}{ccc}
V_{u d} & V_{u s} & V_{u b} \\
V_{c d} & V_{c s} & V_{c b} \\
V_{t d} & V_{t s} & V_{t b}
\end{array}\right)=\left(\begin{array}{ccc}
1-\frac{\lambda^{2}}{2} & \lambda & A \lambda^{3}(\rho-i \eta) \\
-\lambda & 1-\frac{\lambda^{2}}{2} & A \lambda^{2} \\
A \lambda^{3}(1-\rho-i \eta) & -A \lambda^{2} & 1
\end{array}\right),
$$


with the parameters $\lambda=0.2196 \pm 0.0023, A=0.819 \pm 0.035$, and $R_{b} \equiv$ $\sqrt{\rho^{2}+\eta^{2}}=0.41 \pm 0.07$. The unitarity angle $\phi_{3}$ is defined via

$$
V_{u b}=\left|V_{u b}\right| \exp \left(-i \phi_{3}\right) \text {. }
$$

One of the important missions of $B$ fatories is to determine the angles $\phi_{1}$, $\phi_{2}$ and $\phi_{3}$. The angle $\phi_{1}$ can be extracted from the CP asymmetry in the $B \rightarrow$ $J / \psi K_{S}$ decays, which arises from the $B-\bar{B}$ mixing. Due to similar mechanism of CP asymmetry, the decays $B^{0} \rightarrow \pi^{+} \pi^{-}$are appropriate for the extraction of the angle $\phi_{2}$. However, these modes contain penguin contributions such that the extraction suffers large uncertainty. It has been popposed that the angle $\phi_{3}$ can be determined from the decays $B \rightarrow K \pi, \pi \pi$, 迎 6 . Contributions to these modes involve interference between penguin and tree amplitudes, and their analyses rely heavily on theoretical formulations.

\section{Factorization Assumption}

The conventional approach to exclusive nonleptonic $B$ meson decays is based on FAl, in which nonfactorizable and annihilation contributions are neglected and final-state-interaction (FSI) effects are assumed to be absent. Factorizable contributions are expressed as products of Wilson coefficients and hadronic form factors, which are then parametrized by models. With these approximations, the FA approach is simple and provides qualitative estimation of various decay branching ratios.

To extract the angle $\phi_{3}$, we consider the ratios $R$ and $R_{\pi}$ defined by

$$
R=\frac{\operatorname{Br}\left(B_{d}^{0} \rightarrow K^{ \pm} \pi^{\mp}\right)}{\operatorname{Br}\left(B^{ \pm} \rightarrow K^{0} \pi^{ \pm}\right)}, R_{\pi}=\frac{\operatorname{Br}\left(B_{d}^{0} \rightarrow K^{ \pm} \pi^{\mp}\right)}{\operatorname{Br}\left(B_{d}^{0} \rightarrow \pi^{ \pm} \pi^{\mp}\right)}
$$

where $\operatorname{Br}\left(B_{d}^{0} \rightarrow K^{ \pm} \pi^{\mp}\right)$ represents the $\mathrm{CP}$ average of the branching ratios $\operatorname{Br}\left(B_{d}^{0} \rightarrow K^{+} \pi^{-}\right)$and $\operatorname{Br}\left(\bar{B}_{d}^{0} \rightarrow K^{-} \pi^{+}\right)$, and the definition of $\operatorname{Br}\left(B^{ \pm} \rightarrow\right.$ $K^{0} \pi^{ \pm}$) is similar. It has been shown that the data $R \sim 1$ imply $\phi_{3} \sim 90$ 多.

To explain the data of $R_{\pi} \sim 4$, a large angle $\phi_{3} \sim 130^{\circ}$ must be postulated). It is easy to observe from Eqs. (2) and (??) that the products of the CKM matrix elements $V_{u s}^{*} V_{u b}$ and $V_{u d}^{*} V_{u b}$ have the same weak phase, and that the real parts of $V_{t s}^{*} V_{t b}$ and $V_{t d}^{*} V_{u b}$ are opposite in sign. That is, the tree-penguin interference in the decays $B \rightarrow K \pi$ and $B \rightarrow \pi \pi$ is anticorrelated. A $\phi_{3}>90^{\circ}$ then leads to constructive interference between the tree and penguin contributions in $B \rightarrow K \pi$, and a large $R_{\pi}$. The determination $\phi_{3} \sim 114^{\circ}$ from the global fit to charmless $B$ meson decaysl, located between the two extreme cases $90^{\circ}$ and $130^{\circ}$, is then understood. On the other hand, a large $B \rightarrow \rho$ form factor $A_{0}^{B \rho} \sim 0.48$ has been extracted, which 
accounts for the large $B \rightarrow \rho \pi$ branching ratios. In the modified FA approach with an effective number of colors $N_{c}^{\text {eff }}$, a large unitarity angle $\phi_{3} \sim 105^{\circ}$ is also concluded 9 .

The above $\phi_{3}$, located in the second quadrant, contradict the extraction from other measurements, such as $B_{s}$-mixing. The best fit to experimental data of semileptonic $B$ meson decays, $B$ - $\bar{B}$ mixing, and $\epsilon_{K}$ indicates that $\phi_{3}$ is located in the first quadrant. An improvement of FA has been considered, in which possible strong phases produced via FSI are introduced as arbitrary parameter 10 . Performing the best fit to data, a large $\phi_{3}$ is still required and strong phases are found to be large, which generate significant CP asymmetries in $K \pi$ and $\pi \pi$ modes.

\section{$3 \quad S U(3)$ Symmetry}

The model-dependent determination of the angle $\phi_{3}$ from FA seems not to be satisfactory. A more model-independent approach based on $S U(3)$ symmetry has been proposed11, in which the light quarks $u, d$ and $s$ form a $S U(3)$ triplet, while the heavy quarks $c, b$, and $t$ form $S U(3)$ singlets. According to the above assumption, the $B$ mesons $B_{u}, B_{d}$, and $B_{s}$ form a $S U(3)$ triplet at the hadronic level. Pseudo-scalar mesons $P$ and vector mesons $V$ also possess definite $S U(3)$ structures,

$$
\begin{aligned}
& M_{j}^{i}=\left(\begin{array}{ccc}
\frac{\pi^{0}}{\sqrt{2}}-\frac{\eta_{8}}{\sqrt{6}}+\frac{\eta_{1}}{\sqrt{3}} & \pi^{-} & K^{-} \\
\pi^{+} & -\frac{\pi^{0}}{\sqrt{2}}-\frac{\eta_{8}}{\sqrt{6}}+\frac{\eta_{1}}{\sqrt{3}} & \bar{K}^{0} \\
K^{+} & K^{0} & -\frac{2 \eta_{8}}{\sqrt{6}}+\frac{\eta_{1}}{\sqrt{3}}
\end{array}\right), \\
& V_{j}^{i}=\left(\begin{array}{ccc}
\frac{\rho^{0}}{\sqrt{2}}+\frac{\omega}{\sqrt{2}} & \rho^{-} & K^{*-} \\
\rho^{+} & -\frac{\rho^{0}}{\sqrt{2}}+\frac{\omega}{\sqrt{2}} & \bar{K}^{* 0} \\
K^{*+} & K^{* 0} & \phi
\end{array}\right) .
\end{aligned}
$$

Similarly, the four-fermion operators in the weak Hamiltonian can be decomposed into operators with definite $S U(3)$ structures. For example, the penguin operators $O_{3-6}$ are labelled as $\overline{3}$ states,

$$
\bar{q} b(\bar{u} u+\bar{d} d+\bar{s} s) \leftrightarrow \overline{3},
$$

since $\bar{q} b$ forms a triplet $\overline{3}$ and $\bar{u} u+\bar{d} d+\bar{s} s$ froms a singlet. The operators $O_{1,2}$ are written as

$$
\bar{q} u \bar{u} b \leftrightarrow \overline{3} \times 3 \times \overline{3}=\overline{3}+\overline{3}+6+\overline{15} .
$$

Following Eqs. (7) and (8), the effective Hamiltonian in Eq. (1) is decomposed into operators carrying different $S U(3)$ structures, such as $H(\overline{3}), H(6)$ and

ppp5p: submitted to World Scientific on October 30, 2018 
$H(\overline{15})$, whose coefficients are the linear combinations of the Wilson coefficients.

Employing the above results, we formulate various decay amlitudes of the tree and annihilation topologies for $B \rightarrow P P$ modes. For example, the parameter $C_{\overline{3}}$ associated with the contraction $B_{i} M_{k}^{i} M_{j}^{k} H(\overline{3})^{j}$ represents a tree amplitude. The parameter $A_{\overline{3}}$ associated with the contraction $B_{i} H(\overline{3})^{i} M_{l}^{k} M_{k}^{l}$ represents an annihilation amplitude. Collecting all expressions for the branching ratios and the $\mathrm{CP}$ asymmetries of the $B \rightarrow \pi \pi, K \pi$ and $K K$ modes, there are totally 13 free parameters. This number is too big for a global analysis of currently available data. As an approximation, annihilation contributions are neglected. 8 parameters, the absolute values of $C_{\overline{3}}^{T}, C_{\overline{3}}^{P}, C_{6}^{T}$ and $C_{\overline{1} 5}^{T}$, the phases $\delta_{\overline{3}}^{P}, \delta_{6}^{T}$, and $\delta_{\overline{1}}^{T}$, and the CKM phase $\phi_{3}$, are then left, where $T(P)$ denotes the tree operators $O_{1,2}$ (the penguin operators $O_{3-10}$ ).

The best fit to data gives

$$
\begin{aligned}
& \phi_{3}=70^{\circ}, \quad \rho=0.17, \quad \eta=0.37, \\
& C_{\overline{3}}^{T}=0.28, \quad C_{\overline{3}}^{P}=0.14, \quad C_{6}^{T}=0.33, \quad C_{\overline{1} 5}^{T}=0.14, \\
& \delta_{\overline{3}}^{P}=12^{o}, \quad \delta_{6}^{T}=6^{o}, \quad \delta_{\overline{1} 5}^{T}=74^{o} .
\end{aligned}
$$

If the $S U(3)$ symmetry breaking effect from $f_{K} / f_{\pi} \neq 1$ is taken into account, $f_{K}\left(f_{\pi}\right)$ being the kaon (pion) decay constant, the results are shifted only a bit. Hence, there is no indication that $\phi_{3}$ should be located in the second quadrant. Certainly, the allowed range of the above parameters is still large.

\section{Perturbative QCD}

It has been shown that the decay amplitudes and strong phases discussed in the previous sections can be evaluated in the PQCD framework, and that it is possible to extract $\phi_{3}$ from the $B \rightarrow K \pi$ data12. According to PQCD factorization theorem, a $B$ meson decay amplitude is expressed as convolution of a hard $b$ quark decay amplitude with meson wave functions. A meson wave function, absorbing nonperturbative dynamics of a QCD process, is not calculable, while a hard amplitude is.

In perturbation theory nonperturbative dynamics is reflected by infrared divergences in radiative corrections. It has been proved to all orders that these infrared divergences can be separated and absorbed into meson wave functions 13 . A formal definition of wave functions as matrix elements of nonlocal operators has been constructed, which, if evaluated perturbatively, reproduces the infrared divergences. The gauge invariance of the above factorization has been proved in 14 . A meson wave function must be determined by

ppp5p: submitted to World Scientific on October 30, 2018 
nonperturbative means, such as lattice gauge theory and QCD sum rules, or extracted from experimental data. A salient feature of PQCD factorization theorem is the universality of nonperturbative wave functions. Because of universality, a $B$ meson wave function extracted from some decay modes can be employed to make predictions for other modes. This is the reason PQCD factorization theorem possesses a predictive power.

In the practical calculation small parton transverse momenta $k_{T}$ are included 15, which are essential for smearing the end-point singularities from small momentum fractions 12 . Because of the inclusion of $k_{T}$, double logarithms $\ln ^{2}(\mathrm{~Pb})$ are generated from the overlap of collinear and soft enhancements in radiative corrections to meson wave functions, where $P$ denotes the dominant light-cone component of a meson momentum and $b$ is a variable conjugate to $k_{T}$. The resummation of these double logarithms leads to a Sudakov form factor $\exp [-s(P, b)] 16,17$, which suppresses the long-distance contributions in the large $b$ region, and vanishes as $b=1 / \Lambda, \Lambda \equiv \Lambda_{\mathrm{QCD}}$ being the QCD scale. This suppression guarantees the applicability of PQCD to exclusive decays around the energy scale of the $b$ quark mass 18

The hard amplitude contains all possible Feynman diagrams 19 , 20, such as factorizable diagrams, where hard gluons attach the valence quarks in the same meson, and nonfactorizable diagrams, where hard gluons attach the valence quarks in different mesons. The annihilation topology is also included, and classified into factorizable or nonfactorizable one. Therefore, FA for twobody $B$ meson decays is not necessary. It has been shown that factorizable annihilation contributions are in fact important, and give large strong phases in PQCD12.

We emphasize that the hard amplitude is characterized by the virtuality of internal particles, $t \sim \sqrt{\bar{\Lambda} M_{B}} \sim 1.5 \mathrm{GeV}, \bar{\Lambda}=M_{B}-m_{b}$. The RG evolution of the Wilson coefficients $C_{4,6}(t)$ dramatically increase as $t<M_{B} / 2$, such that penguin contributions are enhanced 12,21. With this penguin enhancement, the observed branching ratios of the $B \rightarrow K \pi$ and $B \rightarrow \pi \pi$ decays can be explained in PQCD using a smaller angle $\phi_{3}=90^{\circ}$. That is, the data of $R_{\pi}$ do not demand large $\phi_{3}$. Such a dynamical enhancement of penguin contributions does not exist in the FA approach.

Our predictions for the branching ratio of each $K \pi$ mode corresponding to $\phi_{3}=90$ 12,

$$
\begin{aligned}
& \operatorname{Br}\left(B^{+} \rightarrow K^{0} \pi^{+}\right)=21.72 \times 10^{-6}, \quad \operatorname{Br}\left(B^{-} \rightarrow \bar{K}^{0} \pi^{-}\right)=21.25 \times 10^{-6}, \\
& \operatorname{Br}\left(B_{d}^{0} \rightarrow K^{+} \pi^{-}\right)=24.19 \times 10^{-6}, \quad \operatorname{Br}\left(\bar{B}_{d}^{0} \rightarrow K^{-} \pi^{+}\right)=16.84 \times 10^{-6}, \\
& \operatorname{Br}\left(B^{+} \rightarrow K^{+} \pi^{0}\right)=14.44 \times 10^{-6}, \quad \operatorname{Br}\left(B^{-} \rightarrow K^{-} \pi^{0}\right)=10.65 \times 10^{-6}, \\
& \operatorname{Br}\left(B_{d}^{0} \rightarrow K^{0} \pi^{0}\right)=11.23 \times 10^{-6}, \quad \operatorname{Br}\left(\bar{B}_{d}^{0} \rightarrow \bar{K}^{0} \pi^{0}\right)=11.84 \times 10^{-6},(10)
\end{aligned}
$$

ppp5p: submitted to World Scientific on October 30, 2018 
are consistent with the CLEO data22,

$$
\begin{aligned}
& \operatorname{Br}\left(B^{ \pm} \rightarrow K^{0} \pi^{ \pm}\right)=\left(18.2_{-4.0}^{+4.6} \pm 1.6\right) \times 10^{-6}, \\
& \operatorname{Br}\left(B_{d}^{0} \rightarrow K^{ \pm} \pi^{\mp}\right)=\left(17.2_{-2.4}^{+2.5} \pm 1.2\right) \times 10^{-6}, \\
& \operatorname{Br}\left(B^{ \pm} \rightarrow K^{ \pm} \pi^{0}\right)=\left(11.6_{-2.7-1.3}^{+3.0+1.4}\right) \times 10^{-6}, \\
& \operatorname{Br}\left(B_{d}^{0} \rightarrow K^{0} \pi^{0}\right)=\left(14.6_{-5.1-3.3}^{+5.9+2.4}\right) \times 10^{-6}, \\
& A_{C P}\left(B_{d}^{0} \rightarrow K^{ \pm} \pi^{\mp}\right)=-0.04 \pm 0.16, \\
& A_{C P}\left(B^{ \pm} \rightarrow K^{0} \pi^{ \pm}\right)=0.17 \pm 0.24 .
\end{aligned}
$$

In the above expressions $B\left(B_{d}^{0} \rightarrow K^{ \pm} \pi^{\mp}\right)$ represents the $\mathrm{CP}$ average of the branching ratios $B\left(B_{d}^{0} \rightarrow K^{+} \pi^{-}\right)$and $B\left(\bar{B}_{d}^{0} \rightarrow K^{-} \pi^{+}\right)$.

\section{QCD Factorization}

Recently, Beneke, Buchalla, Neubert, and Sachrajda proposed the QCDF formalism for two-body nonleptonic $B$ meson decays 23 . They claimed that factorizable contributions, for example, the form factor $F^{B \pi}$ in the $B \rightarrow \pi \pi$ decays, are not calculable in PQCD, but nonfactorzable contributions are in the heavy quark limit. Hence, the former are treated in the same way as FA, and expressed as products of Wilson coefficients and $F^{B \pi}$. The latter, calculated as in the PQCD approach, are written as the convolutions of hard amplitudes with three $(B, \pi, \pi)$ meson wave functions. Annihilation diagrams are neglected as in FA, but can be included as $1 / M_{B}$ correction. Values of form factors at maximal recoil and nonperturbative meson wave functions are treated as free parameters.

Here I mention some essential differences between the QCDF and PQCD approaches. For more detailed comparisions, refer to 24 . Because of the neglect of annihilation diagrams in QCDF, strong phases and CP asymmetries are much smaller than those predicted in PQCD. In QCDF the leading-order diagrams are those that contain vertex corrections to the four-fermion operators. These diagrams, however, appear at the next-to-leading order in PQCD. This difference implies different characteristic scales in the two approaches: the former is characterized by the $b$ quark mass $m_{b}$, while the latter is characterized by the virtuality $t$ of internal particles, which leads to the penguin enhancement emphasized above. Without penguin enhancement, a large $\phi_{3}$ is still necessary to account for the large ratio $R_{\pi} 25$.

The $B \rightarrow \phi K$ decays have been analyzed in the QCDF formalism26 27, and branching ratios much smaller than experimental data have been obtained. Since these modes are dominated by penguin contributions, the penguin en-

ppp5p: submitted to World Scientific on October 30, 2018 
hancement may be crucial for explaining the data.

\section{Light-front QCD}

The evaluation of a form factor is simple in the LFQCD formalism, which is written as an overlap integral of initial- and final-state meson wave functions 28. Various form factors have been computed, suchas the $B \rightarrow \pi$, $\rho, K$ and $K^{*}$ form factors 29 and the $\Lambda_{b} \rightarrow \Lambda$ form factors 30 . The results have been employed to predict the decay spectra of the $B \rightarrow K \mu \mu(\tau \tau)$ and $\Lambda_{b} \rightarrow \Lambda \mu \mu(\tau \tau)$ modes. This formalism has been also applied to the radiative leptonic $B$ meson decays $B \rightarrow l^{+} l^{-} \gamma$ 31. These predictions can be compared with data in the future.

\section{Conclusion}

In this talk I have briefly summarized the theoretical progresses on exclusive $B$ meson decays, which were made by Taiwan BCP community recently. With the active collaboration, more progresses are expected in the near future.

\section{Acknowledgments}

This work was supported in part by the National Science Council of R.O.C. under the Grant No. NSC-89-2112-M-006-033, and in part by Grant-in Aid for Special Project Research (Physics of CP Violation) and by Grant-in Aid

for Scientific Exchange from Ministry of Education, Science and Culture of Japan.

\section{References}

1. G. Buchalla, A. J. Buras and M. E. Lautenbacher, Review of Modern Physics 68, 1125 (1996).

2. Review of Particle Physics, Eur. Phys. J. C 3, 1 (1998).

3. M. Gronau, J.L. Rosner, and D. London, Phys. Rev. Lett. 73, 21 (1994); R. Fleischer, Phys. Lett. B 365, 399 (1996).

4. R. Fleischer and T. Mannel, Phys. Rev. D 57, 2752 (1998).

5. M. Neubert and J. Rosner, Phys. Lett. B 441, 403 (1998); M. Neubert, J. High Energy Phys. 02, 14 (1999).

6. A.J. Buras and R. Fleischer, Eur. Phys. J. C 11, 93 (1999).

7. M. Bauer, B. Stech, M. Wirbel, Z. Phys. C 34, 103 (1987); Z. Phys. C 29, 637 (1985). 
8. N.G. Deshpande, X.G. He, W.S. Hou and, S. Pakvasa, Phys. Rev. Lett. 82, 2240 (1999); W.S. Hou, J.G. Smith, and F. Würthwein, hepex/9910014.

9. H.Y. Cheng, hep-ph/9912372; H.Y. Cheng and K.C. Yang, hepph/9910291.

10. W.S. Hou and K.C. Yang, Phys. Rev. Lett. 84, 4806 (2000).

11. X.G. He, Eur. Phys. J. C 9, 443 (1999); N.G. Deshpande, X.G. He, and J.Q. Shi, Phys. Rev. D 62, 034018 (2000).

12. Y.Y. Keum, H-n. Li, and A.I. Sanda, hep-ph/0004004 hep-ph/0004173.

13. H-n. Li, hep-ph/0012140.

14. H.Y. Cheng, H-n. Li, and K.C. Yang, Phys. Rev. D 60, 094005 (1999).

15. H-n. Li and G. Sterman Nucl. Phys. B381, 129 (1992).

16. J.C. Collins and D.E. Soper, Nucl. Phys. B193, 381 (1981).

17. J. Botts and G. Sterman, Nucl. Phys. B325, 62 (1989).

18. H-n. Li and H.L. Yu, Phys. Rev. Lett. 74, 4388 (1995); Phys. Lett. B 353, 301 (1995); Phys. Rev. D 53, 2480 (1996).

19. C.H. Chang and H-n. Li, Phys. Rev. D 55, 5577 (1997).

20. T.W. Yeh and H-n. Li, Phys. Rev. D 56, 1615 (1997).

21. C. D. Lü, K. Ukai, and M. Z. Yang, hep-ph/0004213.

22. CLEO Coll., Y. Kwon et al., hep-ex/9908039.

23. M. Beneke, G. Buchalla, M. Neubert, and C.T. Sachrajda, Phys. Rev. Lett. 83, 1914 (1999); hep-ph/0006124.

24. Y.Y. Keum and H-n. Li, hep-ph/0006001.

25. D. Du, D. Yang, and G. Zhu, hep-ph/0005006; T. Muta, A. Sugamoto, M.Z. Yang, and Y.D. Yang, hep-ph/0006022.

26. H.Y. Cheng and K.C. Yang, hep-ph/0012152.

27. X.G. He, J.P. Ma, and C.Y. Wu, hep-ph/0008159.

28. H.Y. Cheng, C.Y. Cheung, C.W. Hwang, and W.M. Zhang, Phys. Rev. D 57, 5598 (1998).

29. C.Y. Cheung, C.W. Hwang, W.M. Zhang, Z. Phys. C 75, 657 (1997); H.Y. Cheng, C.Y. Cheung, and C.W. Hwang, Phys. Rev. D 55, 1559 (1997).

30. C.H. Chen and C.Q. Geng, hep-ph/0012003.

31. C.Q. Geng, C.C. Lih, and W.M. Zhang, Phys. Rev. D 62, 074017 (2000).

ppp5p: submitted to World Scientific on October 30, 2018 